\title{
Extremal Inverse Eigenvalue Problem for a Special Kind of Matrices
}

\author{
Zhibing Liu, ${ }^{1}$ Yeying Xu, ${ }^{2}$ Kanmin Wang, ${ }^{1}$ and Chengfeng $X u^{1}$ \\ ${ }^{1}$ School of Sciences, Jiujiang University, Jiujiang 332005, China \\ ${ }^{2}$ Faculty of Library, Jiujiang University, Jiujiang 332005, China \\ Correspondence should be addressed to Zhibing Liu; liuzhibingjju@126.com
}

Received 11 June 2013; Accepted 13 December 2013; Published 5 February 2014

Academic Editor: Hui-Shen Shen

Copyright (C) 2014 Zhibing Liu et al. This is an open access article distributed under the Creative Commons Attribution License, which permits unrestricted use, distribution, and reproduction in any medium, provided the original work is properly cited.

We consider the following inverse eigenvalue problem: to construct a special kind of matrix (real symmetric doubly arrow matrix) from the minimal and maximal eigenvalues of all its leading principal submatrices. The necessary and sufficient condition for the solvability of the problem is derived. Our results are constructive and they generate algorithmic procedures to construct such matrices.

\section{Introduction}

Peng et al. in [1] solved two inverse eigenvalue problems for symmetric arrow matrices and, in the other article [2], a correction, for one of the problems stated in [1], has been presented as well. In recent paper [3], Nazari and Beiranvand introduced an algorithm to construct symmetric quasiantibidiagonal matrices that having its given eigenvalues. Pickmann et al. in [4] introduced an algorithm for inverse eigenvalue problem on symmetric tridiagonal matrices. In this paper we introduced symmetric doubly arrow matrix as follows:

$$
A=\left(\begin{array}{ccccccc}
a_{1} & b_{1} & \cdots & b_{s-1} & 0 & \cdots & 0 \\
c b_{1} & a_{2} & \cdots & 0 & 0 & \cdots & 0 \\
\vdots & \vdots & \ddots & 0 & 0 & \cdots & 0 \\
b_{s-1} & 0 & \cdots & a_{s} & b_{s} & \cdots & b_{n-1} \\
0 & 0 & \cdots & b_{s} & a_{s+1} & \cdots & 0 \\
\vdots & \vdots & \cdots & \vdots & \vdots & \ddots & \vdots \\
0 & 0 & 0 & b_{n-1} & 0 & \cdots & a_{n}
\end{array}\right), \quad a_{j}, b_{j} \in \mathbb{R}
$$

where $b_{j} \geq 0,1 \leq s \leq n$. If $s=1$ or $s=n$; then the matrix $A$ of the form (1) is a symmetric arrow matrix as follows:

$$
B=\left(\begin{array}{cccc}
a_{1} & b_{1} & \cdots & b_{n-1} \\
b_{1} & a_{2} & \cdots & 0 \\
\vdots & \vdots & \ddots & \vdots \\
b_{n-1} & 0 & \cdots & a_{n}
\end{array}\right), \quad a_{j}, b_{j} \in \mathbb{R}
$$

This family of matrices appears in certain symmetric inverse eigenvalue and inverse Sturm-Liouville problems $[5,6]$, which arise in many applications [7-12], including modern control theory and vibration analysis $[7,8]$. In this paper, we construct matrix $A$ of the form (1), from a special kind of spectral information, which only recently is being considered. Since this type of matrix structure generalizes the well-known arrow matrices, we think that it will also become of interest in applications.

We will denote $I_{j}$ as the identity matrix of order $j ; A_{j}$ as the $j \times j$ leading principal submatrix of $A ; P_{j}(\lambda)$ as the characteristic polynomial of $A_{j}$; and $\lambda_{1}^{(j)} \leq \lambda_{2}^{(j)} \leq \cdots \leq \lambda_{j}^{(j)}$ as the eigenvalues of $A_{j}$.

We want to solve the following problem. 
Problem 1. Given the $2 n-1$ real numbers $\lambda_{1}^{(j)}$ and $\lambda_{j}^{(j)}$, $j=1,2, \ldots, n$, find an $n \times n$ matrix $A$ of the form (1) such that $\lambda_{1}^{(j)}$ and $\lambda_{j}^{(j)}$ are, respectively, the minimal and maximal eigenvalues of $A_{j}, j=1,2, \ldots, n$.

Our work is motivated by the results in [2]. There, the authors solved this kind of inverse eigenvalue problem for symmetric arrow matrix $B$ of the form (2).

Theorem 2 (see [2]). Let the real numbers $\lambda_{1}^{(j)}$ and $\lambda_{j}^{(j)}, j=$ $1,2, \ldots, n$, be given. Then there exists an $n \times n$ matrix $B$ of the form (2), such that $\lambda_{1}^{(j)}$ and $\lambda_{j}^{(j)}$ are, respectively, the minimal and maximal eigenvalues of its leading principal submatrix $B_{j}$, $j=1,2, \ldots, n$, if and only if

$$
\lambda_{1}^{(n)} \leq \cdots \leq \lambda_{1}^{(3)} \leq \lambda_{1}^{(2)} \leq \lambda_{1}^{(1)} \leq \lambda_{2}^{(2)} \leq \lambda_{3}^{(3)} \leq \cdots \leq \lambda_{n}^{(n)} .
$$

Theorem 3 (see [2]). Let the real numbers $\lambda_{1}^{(j)}$ and $\lambda_{j}^{(j)}, j=$ $1,2, \ldots, n$, be given. Then there exists a unique $n \times n$ matrix $B$ of the form (2), with $a_{j} \in \mathbb{R}$ and $b_{j}>0$, such that $\lambda_{1}^{(j)}$ and $\lambda_{j}^{(j)}$ are, respectively, the minimal and the maximal eigenvalues of its leading principal submatrix $B_{j}, j=1,2, \ldots, n$, if and only if

$$
\lambda_{1}^{(n)}<\cdots<\lambda_{1}^{(3)}<\lambda_{1}^{(2)}<\lambda_{1}^{(1)}<\lambda_{2}^{(2)}<\lambda_{3}^{(3)}<\cdots<\lambda_{n}^{(n)} .
$$

In this paper, we will show that Theorems 2 and 3 are also right for symmetric doubly arrow matrix $A$ in (1) by a similar method.

The paper is organized as follows. In Section 2, we discuss some properties of $A$. In Section 3, we solve Problem 1 by giving a necessary and sufficient condition for the existence of the matrix $A$ in (1) and also solve the case in which the matrix $A$, in Problem 1 , is required to have all its entries $b_{i}$ positive. Finally, In Section 4 we show some examples to illustrate the results.

\section{Properties of the Matrix $A$}

Lemma 4. Let $A$ be a matrix of the form (1). Then the sequence of characteristic polynomials $\left\{P_{j}(\lambda)\right\}_{j=1}^{n}$ satisfies the recurrence relation:

$$
\begin{gathered}
p_{1}(\lambda)=\left(\lambda-a_{1}\right), \\
p_{j}(\lambda)=\left(\lambda-a_{j}\right) p_{j-1}(\lambda)-b_{j-1}^{2} \prod_{i=2}^{j-1}\left(\lambda-a_{i}\right), \\
j=2,3, \ldots, s, \\
p_{j}(\lambda)=\left(\lambda-a_{j}\right) p_{j-1}(\lambda)-b_{j-1}^{2} \prod_{i=s+1}^{j-1}\left(\lambda-a_{i}\right) p_{s-1}(\lambda), \\
j=s+1, \ldots, n .
\end{gathered}
$$

Proof. It is easy to verify by expanding the determinant.
Lemma 5 (see [2]). Let $p(\lambda)$ be a monic polynomial of degree $n$ with all real zeroes. If $\lambda_{1}$ and $\lambda_{n}$ are, respectively, the minimal and maximal zeroes of $p(\lambda)$, then

(1) if $\mu<\lambda_{1}$, we have that $(-1)^{n} p(\mu)>0$;

(2) if $\mu>\lambda_{n}$, we have that $p(\mu)>0$.

Observe that, from the Cauchy interlacing property, the minimal and the maximal eigenvalue, $\lambda_{1}^{(j)}$ and $\lambda_{j}^{(j)}$, respectively, of each leading principal submatrix $A_{j}, j=1,2, \ldots, n$, of the matrix $A$ in (1) satisfy the relations

$$
\begin{gathered}
\lambda_{1}^{(n)} \leq \cdots \leq \lambda_{1}^{(3)} \leq \lambda_{1}^{(2)} \leq \lambda_{1}^{(1)} \leq \lambda_{2}^{(2)} \leq \lambda_{3}^{(3)} \leq \cdots \leq \lambda_{n}^{(n)}, \\
\lambda_{1}^{(j)} \leq a_{i} \leq \lambda_{j}^{(j)}, \quad i=1,2, \ldots, j ; j=1,2, \ldots, n .
\end{gathered}
$$

Lemma 6. Let $\left\{P_{j}(\lambda)\right\}_{j=1}^{n}$ be the polynomials defined in (5), whose minimal and maximal zeroes, $\lambda_{1}^{(j)}$ and $\lambda_{j}^{(j)}, j=$ $1,2, \ldots, n$, respectively, satisfy the relations (6) and (7), and $h_{j}$

$$
=\left\{\begin{array}{c}
p_{j-1}\left(\lambda_{1}^{(j)}\right) \prod_{i=2}^{j-1}\left(\lambda_{j}^{(j)}-a_{i}\right)-p_{j-1}\left(\lambda_{j}^{(j)}\right) \prod_{i=2}^{j-1}\left(\lambda_{1}^{(j)}-a_{i}\right), \\
p_{j-1}\left(\lambda_{1}^{(j)}\right) p_{s-1}\left(\lambda_{j}^{(j)}\right) \\
\quad \times \prod_{i=s+1}^{j-1}\left(\lambda_{j}^{(j)}-a_{i}\right)-p_{j-1}\left(\lambda_{j}^{(j)}\right) p_{s-1}\left(\lambda_{1}^{(j)}\right) \\
\times \prod_{i=s+1}^{j-1}\left(\lambda_{1}^{(j)}-a_{i}\right), \quad j=s+1, \ldots, n .
\end{array}\right.
$$

Then

$$
\tilde{h}_{j}=(-1)^{j-1} h_{j} \geq 0, \quad j=2,3, \ldots, n .
$$

Proof. From Lemma 5, we have

$$
\begin{array}{rr}
(-1)^{j-1} p_{j-1}\left(\lambda_{1}^{(j)}\right) \geq 0, & p_{j-1}\left(\lambda_{j}^{(j)}\right) \geq 0, \\
& j=2,3, \ldots, n, \\
(-1)^{s-1} p_{s-1}\left(\lambda_{1}^{(j)}\right) \geq 0, & p_{s-1}\left(\lambda_{j}^{(j)}\right) \geq 0, \\
& j=s+1, \ldots, n .
\end{array}
$$

Moreover, from (7)

$$
\begin{array}{r}
\prod_{i=2}^{j-1}\left(\lambda_{j}^{(j)}-a_{i}\right) \geq 0, \quad(-1)^{j} \prod_{i=2}^{j-1}\left(\lambda_{1}^{(j)}-a_{i}\right) \geq 0, \\
j=2,3, \ldots, s, \\
\prod_{i=s+1}^{j-1}\left(\lambda_{j}^{(j)}-a_{i}\right) \geq 0, \quad(-1)^{j-s+1} \prod_{i=s+1}^{j-1}\left(\lambda_{1}^{(j)}-a_{i}\right) \geq 0, \\
j=s+1, \ldots, n .
\end{array}
$$


Lemma 7 (see [2]). Let $A$ be a matrix of the form (2) with $b_{i} \neq 0, i=1,2, \ldots, n-1$. Let $\lambda_{1}^{(j)}$ and $\lambda_{j}^{(j)}$, respectively, be the minimal and maximal eigenvalues of the leading principal submatrix $A_{j}, j=1,2, \ldots, n$, of $A$. Then

$$
\begin{gathered}
\lambda_{1}^{(j)}<\cdots<\lambda_{1}^{(3)}<\lambda_{1}^{(2)}<\lambda_{1}^{(1)}<\lambda_{2}^{(2)}<\lambda_{3}^{(3)}<\cdots<\lambda_{j}^{(j)}, \\
\lambda_{1}^{(j)}<a_{i}<\lambda_{j}^{(j)}, \quad i=2,3, \ldots, j
\end{gathered}
$$

for each $j=2,3, \ldots, n$.

\section{Solution of Problem 1}

The following theorem solves Problem 1. In particular, the theorem shows that condition (6) is necessary and sufficient for the existence of the matrix $A$ in (1).

Theorem 8. Let the real numbers $\lambda_{1}^{(j)}$ and $\lambda_{j}^{(j)}, j=1,2, \ldots, n$, be given. Then there exists an $n \times n$ matrix $A$ of the form (1), such that $\lambda_{1}^{(j)}$ and $\lambda_{j}^{(j)}$ are, respectively, the minimal and maximal eigenvalues of its leading principal submatrix $A_{j}, j=$ $1,2, \ldots, n$, if and only if

$$
\lambda_{1}^{(n)} \leq \cdots \leq \lambda_{1}^{(3)} \leq \lambda_{1}^{(2)} \leq \lambda_{1}^{(1)} \leq \lambda_{2}^{(2)} \leq \lambda_{3}^{(3)} \leq \cdots \leq \lambda_{n}^{(n)} .
$$

Proof. Let $\lambda_{1}^{(j)}$ and $\lambda_{j}^{(j)}, j=1,2, \ldots, n$, satisfy (13). Observe that

$$
A_{1}=\left(a_{1}\right)=\left(\lambda_{1}^{(1)}\right)
$$

and $p_{1}(\lambda)=\lambda-a_{1}$. From Theorem 2 , there exists $A_{j}, j=2$, $\ldots, s$ with $\lambda_{1}^{(j)}$ and $\lambda_{j}^{(j)}$ as its minimal and maximal eigenvalues, respectively. To show the existence of $A_{j}, j=s+1, \ldots, n$ with $\lambda_{1}^{(j)}$ and $\lambda_{j}^{(j)}$ as its minimal and maximal eigenvalues, respectively, is equivalent to showing that the system of equations

$$
\begin{aligned}
p_{j}\left(\lambda_{1}^{(j)}\right)= & \left(\lambda_{1}^{(j)}-a_{j}\right) p_{j-1}\left(\lambda_{1}^{(j)}\right) \\
& -b_{j-1}^{2} \prod_{i=s+1}^{j-1}\left(\lambda_{1}^{(j)}-a_{i}\right) p_{s-1}\left(\lambda_{1}^{(j)}\right)=0, \\
p_{j}\left(\lambda_{j}^{(j)}\right)= & \left(\lambda_{j}^{(j)}-a_{j}\right) p_{j-1}\left(\lambda_{j}^{(j)}\right) \\
& -b_{j-1}^{2} \prod_{i=s+1}^{j-1}\left(\lambda_{j}^{(j)}-a_{i}\right) p_{s-1}\left(\lambda_{j}^{(j)}\right)=0
\end{aligned}
$$

has real solution $a_{j}$ and $b_{j-1}, j=s+1, \ldots, n$. If the determinant

$$
\begin{aligned}
h_{j}= & p_{j-1}\left(\lambda_{1}^{(j)}\right) p_{s-1}\left(\lambda_{j}^{(j)}\right) \\
& \times \prod_{i=s+1}^{j-1}\left(\lambda_{j}^{(j)}-a_{i}\right)-p_{j-1}\left(\lambda_{j}^{(j)}\right) p_{s-1}\left(\lambda_{1}^{(j)}\right) \\
& \times \prod_{i=s+1}^{j-1}\left(\lambda_{1}^{(j)}-a_{i}\right)
\end{aligned}
$$

of the coefficient matrix of the system (15) is nonzero, then the system has unique solutions $a_{j}$ and $b_{j-1}^{2}, j=s+1, \ldots, n$. In this case, from Lemma 6 we have $\widetilde{h}_{j}>0$. By solving the system (15) we obtain

$$
\begin{aligned}
a_{j}= & \left(\lambda_{1}^{(j)} p_{j-1}\left(\lambda_{1}^{(j)}\right) p_{s-1}\left(\lambda_{j}^{(j)}\right)\right. \\
& \times \prod_{i=s+1}^{j-1}\left(\lambda_{j}^{(j)}-a_{i}\right)-\lambda_{j}^{(j)} p_{j-1}\left(\lambda_{j}^{(j)}\right) p_{s-1}\left(\lambda_{1}^{(j)}\right) \\
& \left.\times \prod_{i=s+1}^{j-1}\left(\lambda_{1}^{(j)}-a_{i}\right)\right)\left(h_{j}\right)^{-1}, \\
b_{j-1}^{2} & =\frac{\left(\lambda_{j}^{(j)}-\lambda_{1}^{(j)}\right) p_{j-1}\left(\lambda_{1}^{(j)}\right) p_{j-1}\left(\lambda_{j}^{(j)}\right)}{h_{j}} .
\end{aligned}
$$

Since

$$
(-1)^{j-1}\left(\lambda_{j}^{(j)}-\lambda_{1}^{(j)}\right) p_{j-1}\left(\lambda_{1}^{(j)}\right) p_{j-1}\left(\lambda_{j}^{(j)}\right) \geq 0 ;
$$

then $b_{j-1}$ is a real number and therefore, there exists $A$ with the spectral properties required.

Now we will show that, if $h_{j}=0$, the system (15) still has a solution. We do this by induction by showing that the rank of the coefficients matrix is equal to the rank of the augmented matrix.

Let $j=s+1$. If $h_{s+1}=0$, then

$$
\begin{aligned}
\tilde{h}_{s+1}= & (-1)^{s} h_{s+1} \\
= & (-1)^{s}\left(p_{s}\left(\lambda_{1}^{(s+1)}\right) p_{s-1}\left(\lambda_{s+1}^{(s+1)}\right)\right. \\
& \left.\quad-p_{s}\left(\lambda_{s+1}^{(s+1)}\right) p_{s-1}\left(\lambda_{1}^{(s+1)}\right)\right)=0,
\end{aligned}
$$

which, from Lemma 5, is equivalent to

$$
\begin{aligned}
& p_{s}\left(\lambda_{1}^{(s+1)}\right) p_{s-1}\left(\lambda_{s+1}^{(s+1)}\right)=0, \\
& p_{s}\left(\lambda_{s+1}^{(s+1)}\right) p_{s-1}\left(\lambda_{1}^{(s+1)}\right)=0 .
\end{aligned}
$$

In this case the augmented matrix is

$$
\left(\begin{array}{lll}
p_{s}\left(\lambda_{1}^{(s+1)}\right) & p_{s-1}\left(\lambda_{1}^{(s+1)}\right) & \lambda_{1}^{(s+1)} p_{s}\left(\lambda_{1}^{(s+1)}\right) \\
p_{s}\left(\lambda_{s+1}^{(s+1)}\right) & p_{s-1}\left(\lambda_{s+1}^{(s+1)}\right) & \lambda_{s+1}^{(s+1)} p_{s}\left(\lambda_{s+1}^{(s+1)}\right)
\end{array}\right),
$$


and the ranks of both matrices, the coefficient matrix and the augmented matrix, are equal. Hence $A_{s+1}$ exists.

Now we consider $j \geq s+2$. If $h_{j}=0$, then

$$
\begin{aligned}
\tilde{h}_{j}=(-1)^{j-1} h_{j} & \\
=(-1)^{j-1} & \left(p_{j-1}\left(\lambda_{1}^{(j)}\right) p_{s-1}\left(\lambda_{j}^{(j)}\right)\right. \\
& \times \prod_{i=s+1}^{j-1}\left(\lambda_{j}^{(j)}-a_{i}\right)-p_{j-1}\left(\lambda_{j}^{(j)}\right) p_{s-1}\left(\lambda_{1}^{(j)}\right) \\
& \left.\times \prod_{i=s+1}^{j-1}\left(\lambda_{1}^{(j)}-a_{i}\right)\right)=0 .
\end{aligned}
$$

\section{From Lemma 5}

$$
\begin{aligned}
& p_{j-1}\left(\lambda_{1}^{(j)}\right)=0 \bigvee p_{s-1}\left(\lambda_{j}^{(j)}\right) \prod_{i=s+1}^{j-1}\left(\lambda_{j}^{(j)}-a_{i}\right)=0, \\
& p_{j-1}\left(\lambda_{j}^{(j)}\right)=0 \bigvee p_{s-1}\left(\lambda_{1}^{(j)}\right) \prod_{i=s+1}^{j-1}\left(\lambda_{1}^{(j)}-a_{i}\right)=0 .
\end{aligned}
$$

Then $h_{j}=0$ leads us to the following cases:

(i) $\lambda_{1}^{(j)}=\lambda_{1}^{(j-1)} \wedge \lambda_{j-1}^{(j-1)}=\lambda_{j}^{(j)}$,

(ii) $\lambda_{1}^{(j)}=\lambda_{1}^{(j-1)} \wedge p_{s-1}\left(\lambda_{1}^{(j)}\right) \prod_{i=s+1}^{j-1}\left(\lambda_{1}^{(j)}-a_{i}\right)=0$,

(iii) $p_{s-1}\left(\lambda_{j}^{(j)}\right) \prod_{i=s+1}^{j-1}\left(\lambda_{j}^{(j)}-a_{i}\right)=0 \wedge \lambda_{j-1}^{(j-1)}=\lambda_{j}^{(j)}$,

(iv) $p_{s-1}\left(\lambda_{j}^{(j)}\right) \prod_{i=s+1}^{j-1}\left(\lambda_{j}^{(j)}-a_{i}\right)=0 \wedge p_{s-1}\left(\lambda_{1}^{(j)}\right) \prod_{i=s+1}^{j-1}\left(\lambda_{1}^{(j)}-\right.$ $\left.a_{i}\right)=0$,

and the augmented matrix is

$$
\left(\begin{array}{ccc}
p_{j-1}\left(\lambda_{1}^{(j)}\right) & p_{s-1}\left(\lambda_{1}^{(j)}\right) \prod_{i=s+1}^{j-1}\left(\lambda_{1}^{(j)}-a_{i}\right) & \lambda_{1}^{(j)} p_{j-1}\left(\lambda_{1}^{(j)}\right) \\
p_{j-1}\left(\lambda_{j}^{(j)}\right) & p_{s-1}\left(\lambda_{j}^{(j)}\right) \prod_{i=s+1}^{j-1}\left(\lambda_{j}^{(j)}-a_{i}\right) \lambda_{j}^{(j)} p_{j-1}\left(\lambda_{j}^{(j)}\right)
\end{array}\right) .
$$

By replacing conditions (i)-(iii) in (24), it is clear that the coefficients matrix and the augmented matrix have the same rank. From condition (iv), the system of (15) becomes

$$
\begin{aligned}
& p_{j-1}\left(\lambda_{1}^{(j)}\right) a_{j}=\lambda_{1}^{(j)} p_{j-1}\left(\lambda_{1}^{(j)}\right), \\
& p_{j-1}\left(\lambda_{j}^{(j)}\right) a_{j}=\lambda_{j}^{(j)} p_{j-1}\left(\lambda_{j}^{(j)}\right) .
\end{aligned}
$$

If $p_{j-1}\left(\lambda_{1}^{(j)}\right) \neq 0$ and $p_{j-1}\left(\lambda_{j}^{(j)}\right) \neq 0$, then $a_{j}=\lambda_{1}^{(j)}=\lambda_{j}^{(j)}$ and from (13)

$$
\lambda_{1}^{(j)}=\lambda_{1}^{(j-1)}=\cdots=\lambda_{1}^{(1)}=\cdots=\lambda_{j-1}^{(j-1)}=\lambda_{j}^{(j)} .
$$

Thus, $p_{j-1}\left(\lambda_{1}^{(j)}\right)=p_{j-1}\left(\lambda_{j}^{(j)}\right)=0$, which is a contradiction. Hence, under condition (iv) $p_{j-1}\left(\lambda_{1}^{(j)}\right)=0$ or $p_{j-1}\left(\lambda_{j}^{(j)}\right)=0$ and therefore the coefficients matrix and the augmented matrix have also the same rank. By taking $b_{j-1}^{2} \geq 0$, there exists a $j \times j$ matrix $A_{j}$ with the required spectral properties. The necessity comes from the Cauchy interlacing property.

We have seen in the proof of Theorem 8 that if the determinant $h_{j}$ of the coefficients matrix of the system (15) is nonzero, then the Problem 1 has a unique solution except for the sign of the $b_{i}$ entries.

Now we solve the Problem 1 in the case that the $b_{i}$ entries are required to be positive. We need the following lemma.

Lemma 9. Let $A$ be a matrix of the form (1) with $b_{i} \neq 0, i=$ $1,2, \ldots, n-1$. Let $\lambda_{1}^{(j)}$ and $\lambda_{j}^{(j)}$, respectively, be the minimal and maximal eigenvalues of the leading principal submatrix $A_{j}, j=1,2, \ldots, n$, of $A$. Then

$$
\begin{gathered}
\lambda_{1}^{(j)}<\cdots<\lambda_{1}^{(3)}<\lambda_{1}^{(2)}<\lambda_{1}^{(1)}<\lambda_{2}^{(2)}<\lambda_{3}^{(3)}<\cdots<\lambda_{j}^{(j)}, \\
\lambda_{1}^{(j)}<a_{i}<\lambda_{j}^{(j)}, \quad i=2,3, \ldots, j
\end{gathered}
$$

for each $j=2,3, \ldots, n$.

Proof. From Lemma 7, (27) hold for $j \leq s$. For $j=s+1$, we have from (5)

$$
p_{s+1}(\lambda)=\left(\lambda-a_{s+1}\right) p_{s}-b_{s}^{2} p_{s-1}(\lambda) .
$$

As $b_{s} \neq 0$, then from Lemma 7 , we have $p_{s+1}\left(\lambda_{1}^{(s)}\right) \neq 0$, $p_{s+1}\left(\lambda_{s}^{(s)}\right) \neq 0$, and

$$
\lambda_{1}^{(s+1)}<\lambda_{1}^{(s)}<\cdots<\lambda_{1}^{(2)}<\lambda_{1}^{(1)}<\lambda_{2}^{(2)}<\cdots<\lambda_{s}^{(s)}<\lambda_{s+1}^{(s+1)} .
$$

If $\lambda_{1}^{(s+1)}=a_{s+1}$ or $\lambda_{s+1}^{(s+1)}=a_{s+1}$, then

$$
0=p_{s+1}\left(a_{s+1}\right)=-b_{s}^{2} p_{s-1}\left(a_{s+1}\right)
$$

contradicts $b_{s} \neq 0$ or (29) and from (7) we have

$$
\lambda_{1}^{(s+1)}<a_{s+1}<\lambda_{s+1}^{(s+1)} .
$$

Let $j=s+2$. Then from (5)

$$
p_{s+2}\left(\lambda_{1}^{(s+1)}\right)=-b_{s+1}^{2}\left(\lambda_{1}^{(s+1)}-a_{s+1}\right) p_{s-1}\left(\lambda_{1}^{(s+1)}\right) \neq 0 .
$$

In the same way $p_{s+2}\left(\lambda_{s+1}^{(s+1)}\right) \neq 0$. Hence $\lambda_{1}^{(s+1)}$ and $\lambda_{s+1}^{(s+1)}$ are not zeroes of $p_{s+2}(\lambda)$ and from (6)

$$
\begin{aligned}
\lambda_{1}^{(s+2)} & <\lambda_{1}^{(s+1)}<\lambda_{1}^{(s)}<\cdots<\lambda_{1}^{(2)}<\lambda_{1}^{(1)} \\
& <\lambda_{2}^{(2)}<\cdots<\lambda_{s}^{(s)}<\lambda_{s+1}^{(s+1)}<\lambda_{s+2}^{(s+2)}
\end{aligned}
$$

Now suppose that $\lambda_{1}^{(s+2)}=a_{s+2}$. Then

$$
\begin{aligned}
0 & =p_{s+2}\left(a_{s+2}\right)=-b_{s+1}^{2}\left(a_{s+2}-a_{s+1}\right) p_{s-1}\left(a_{s+2}\right) \\
& =-b_{s+1}^{2}\left(\lambda_{1}^{s+2}-a_{s+1}\right) p_{s-1}\left(a_{s+2}\right)
\end{aligned}
$$


contradicts the inequalities (31) and (33). The same occurs if we assume that $\lambda_{s+2}^{(s+2)}=a_{s+2}$. Then from (7) and Lemma 7 we have

$$
\lambda_{1}^{(s+2)}<a_{i}<\lambda_{s+2}^{(s+2)}, \quad i=2,3, \ldots, s+2 .
$$

Now, suppose that (27) hold for $s+3 \leq j \leq n-1$ and consider

$$
p_{j+1}(\lambda)=\left(\lambda-a_{j+1}\right) p_{j}(\lambda)-b_{j}^{2} \prod_{i=s+1}^{j}\left(\lambda-a_{i}\right) p_{s-1}(\lambda) .
$$

Since $b_{j} \neq 0$ and $\lambda_{1}^{(j)}<a_{i}<\lambda_{j}^{(j)}, i=2,3, \ldots, j$, then $\prod_{i=s+1}^{j}\left(\lambda_{1}^{j}-a_{i}\right) \neq 0$ and $\prod_{i=s+1}^{j}\left(\lambda_{j}^{j}-a_{i}\right) \neq 0$. Hence neither $\lambda_{1}^{j}$ nor $\lambda_{j}^{j}$ are zeroes of $p_{j+1}(\lambda)$. Then from (6) we have

$$
\lambda_{1}^{(j+1)}<\lambda_{1}^{(j)}<\cdots<\lambda_{1}^{(2)}<\lambda_{1}^{(1)}<\lambda_{2}^{(2)}<\cdots<\lambda_{j}^{(j)}<\lambda_{j+1}^{(j+1)} .
$$

Finally, if $\lambda_{1}^{(j+1)}=a_{j+1}$, then

$$
\begin{aligned}
0 & =p_{j+1}\left(a_{j+1}\right)=-b_{j}^{2} \prod_{i=s+1}^{j}\left(a_{j+1}-a_{i}\right) p_{s-1}\left(a_{j+1}\right) \\
& =-b_{j}^{2} \prod_{i=s+1}^{j}\left(\lambda_{1}^{j+1}-a_{i}\right) p_{s-1}\left(\lambda_{1}^{j+1}\right)
\end{aligned}
$$

contradicts (33). Then

$$
\lambda_{1}^{(j+1)}<a_{i}<\lambda_{j+1}^{(j+1)}, \quad i=2,3, \ldots, j+1 .
$$

The following theorem solves Problem 1 with $b_{j}>0$.

Theorem 10. Let the real numbers $\lambda_{1}^{(j)}$ and $\lambda_{j}^{(j)}, j=1,2$, $\ldots, n$, be given. Then there exists a unique $n \times n$ matrix $A$ of the form (1), with $a_{j} \in \mathbb{R}$ and $b_{j}>0$, such that $\lambda_{1}^{(j)}$ and $\lambda_{j}^{(j)}$ are, respectively, the minimal and maximal eigenvalues of its leading principal submatrix $A_{j}, j=1,2, \ldots, n$, if and only if

$$
\lambda_{1}^{(n)}<\cdots<\lambda_{1}^{(3)}<\lambda_{1}^{(2)}<\lambda_{1}^{(1)}<\lambda_{2}^{(2)}<\lambda_{3}^{(3)}<\cdots<\lambda_{n}^{(n)} .
$$

Proof. The proof is quite similar to the proof of Theorem 8: Let $\lambda_{1}^{(j)}$ and $\lambda_{j}^{(j)}, j=2, \ldots, n$, satisfy (40). From Theorem 3 , there exists $A_{j}, j=2, \ldots, s$ with $\lambda_{1}^{(j)}$ and $\lambda_{j}^{(j)}$ as its minimal and maximal eigenvalues, respectively. To show the existence of $A_{j}, j=s+1, \ldots, n$ with the required spectral properties, is equivalent to showing that the system of (15) has real solutions $a_{j}$ and $b_{j-1}$, with $b_{j-1}>0, j=s+1, s+2, \ldots, n$.
To do this it is enough to show that the determinant of the coefficients matrix

$$
\begin{aligned}
h_{j}= & p_{j-1}\left(\lambda_{1}^{(j)}\right) p_{s-1}\left(\lambda_{j}^{(j)}\right) \\
& \times \prod_{i=s+1}^{j-1}\left(\lambda_{j}^{(j)}-a_{i}\right)-p_{j-1}\left(\lambda_{j}^{(j)}\right) p_{s-1}\left(\lambda_{1}^{(j)}\right) \\
& \times \prod_{i=s+1}^{j-1}\left(\lambda_{1}^{(j)}-a_{i}\right)
\end{aligned}
$$

is nonzero.

From Lemmas 6 and 9 it follows that $\widetilde{h}_{j}=(-1)^{j-1} h_{j}>$ 0 . Hence $h_{j} \neq 0$ and the system (15) has real and unique solutions:

$$
\begin{aligned}
a_{j}= & \left(\lambda_{1}^{(j)} p_{j-1}\left(\lambda_{1}^{(j)}\right) p_{s-1}\left(\lambda_{j}^{(j)}\right)\right. \\
& \times \prod_{i=s+1}^{j-1}\left(\lambda_{j}^{(j)}-a_{i}\right)-\lambda_{j}^{(j)} p_{j-1}\left(\lambda_{j}^{(j)}\right) p_{s-1}\left(\lambda_{1}^{(j)}\right) \\
& \left.\times \prod_{i=s+1}^{j-1}\left(\lambda_{1}^{(j)}-a_{i}\right)\right)\left(h_{j}\right)^{-1}, \\
b_{j-1}^{2} & =\frac{\left(\lambda_{j}^{(j)}-\lambda_{1}^{(j)}\right) p_{j-1}\left(\lambda_{1}^{(j)}\right) p_{j-1}\left(\lambda_{j}^{(j)}\right)}{h_{j}}
\end{aligned}
$$

where

$$
(-1)^{j-1}\left(\lambda_{j}^{(j)}-\lambda_{1}^{(j)}\right) p_{j-1}\left(\lambda_{1}^{(j)}\right) p_{j-1}\left(\lambda_{j}^{(j)}\right)>0
$$

Then it is clear that $b_{j-1}^{2}>0$. Therefore, the $b_{j-1}$ can be chosen positive and then there exists a unique matrix $A_{j}$ with the required spectral properties. The necessity of the result comes from Lemma 9.

\section{Examples}

Now we give an algorithm to construct the solution $A$ of Problem 1.

Algorithm.

(1) Input a positive integer $s$ and real numbers $\lambda_{1}^{(j)}$ and $\lambda_{j}^{(j)}, j=1,2, \ldots, n$;

(2) let $a_{1}=\lambda_{1}^{(1)} \cdot p_{1}(\lambda)=\lambda-a_{1}$;

(3) for $j=2, \ldots, n$, calculate $p_{j}(\lambda)$ according to (5);

(4) compute $a_{j}$ and $b_{j-1}$ according to (17). 
Example 1. The following numbers [2]

$$
\begin{array}{cccccc}
\lambda_{1}^{(5)} & \lambda_{1}^{(4)} & \lambda_{1}^{(3)} & \lambda_{1}^{(2)} & \lambda_{1}^{(1)} & \lambda_{2}^{(2)} \\
-11.2369 & -11.1921 & -10.9106 & -8.7760 & -6.0043 & -2.6295 \\
\lambda_{3}^{(3)} & \lambda_{4}^{(4)} & \lambda_{5}^{(5)} & & & \\
1.8532 & 8.4266 & 10.4020 & & &
\end{array}
$$

satisfy the necessary and sufficient condition (40) of Theorem 10. Then the doubly arrow matrix with $b_{i}>0$ and

$$
s=3 \text { is }
$$

$$
A=\left(\begin{array}{ccccc}
-6.0043 & 3.0584 & 5.2453 & & \\
3.0584 & -5.4011 & & & \\
5.2453 & & -2.3357 & 4.4747 & 1.8880 \\
& & 4.4747 & 6.1414 & \\
& & 1.8880 & & 9.8361
\end{array}\right) \text {. }
$$

From the above $5 \times 5$ doubly arrow matrix $A$, we recompute the spectrum $\sigma\left(A_{j}\right)$ of its submatrix $A_{j}$ by MATLAB 7.0, $j=$ $1,2, \ldots, 5$, and get

$$
\begin{aligned}
& \sigma\left(A_{1}\right) \overline{\lambda_{1}^{(1)}}=-6.0043, \\
& \sigma\left(A_{2}\right) \overline{\lambda_{1}^{(2)}}=-8.7759 \overline{\lambda_{2}^{(2)}}=-2.6295, \\
& \sigma\left(A_{3}\right) \overline{\lambda_{1}^{(3)}}=-10.9106 \overline{\lambda_{2}^{(3)}}=-4.6837 \overline{\lambda_{3}^{(3)}}=1.8532, \\
& \sigma\left(A_{4}\right) \overline{\lambda_{1}^{(4)}}=-11.1921 \overline{\lambda_{2}^{(4)}}=-5.0836 \overline{\lambda_{3}^{(4)}}=0.2494 \overline{\lambda_{4}^{(4)}}=8.4266, \\
& \sigma\left(A_{5}\right) \overline{\lambda_{1}^{(5)}}=-11.2369 \overline{\lambda_{2}^{(5)}}=-5.1390 \overline{\lambda_{3}^{(5)}}=0.1384 \overline{\lambda_{4}^{(5)}}=8.0719 \overline{\lambda_{5}^{(5)}}=10.4020 .
\end{aligned}
$$

Example 2. We modify the previous example, that some given eigenvalues become equal to [2]

$$
\begin{array}{cccccc}
\lambda_{1}^{(5)} & \lambda_{1}^{(4)} & \lambda_{1}^{(3)} & \lambda_{1}^{(2)} & \lambda_{1}^{(1)} & \lambda_{2}^{(2)} \\
-11.2369 & -10.9106 & -10.9106 & -8.7760 & -6.0043 & -6.0043 \\
\lambda_{3}^{(3)} & \lambda_{4}^{(4)} & \lambda_{5}^{(5)} & & & \\
1.8532 & 8.4266 & 10.4020 . & & &
\end{array}
$$

matrix

$$
A=\left(\begin{array}{ccccc}
-6.0043 & 0 & 6.2090 & & \\
0 & -8.7760 & & & \\
6.2090 & & -3.0531 & 0 & 4.0562 \\
& & 0 & 8.4266 & \\
& & 4.0562 & & 8.9205
\end{array}\right)
$$

These numbers satisfy the necessary and sufficient condition (13) of Theorem 8. One solution of Problem 1 with $s=3$ is the
Recomputing the spectrum of $A$, we have

$$
\begin{aligned}
& \sigma\left(A_{1}\right) \overline{\lambda_{1}^{(1)}}=-6.0043, \\
& \sigma\left(A_{2}\right) \overline{\lambda_{1}^{(2)}}=-8.7760 \overline{\lambda_{2}^{(2)}}=-6.0043, \\
& \sigma\left(A_{3}\right) \overline{\lambda_{1}^{(3)}}=-10.9106 \overline{\lambda_{2}^{(3)}}=-8.7760 \overline{\lambda_{3}^{(3)}}=1.8532, \\
& \sigma\left(A_{4}\right) \overline{\lambda_{1}^{(4)}}=-10.9106 \overline{\lambda_{2}^{(4)}}=-8.7760 \overline{\lambda_{3}^{(4)}}=1.8532 \overline{\lambda_{4}^{(4)}}=8.4266, \\
& \sigma\left(A_{5}\right) \overline{\lambda_{1}^{(5)}}=-11.2369 \overline{\lambda_{2}^{(5)}}=-8.7760 \overline{\lambda_{3}^{(5)}}=0.6980 \overline{\lambda_{4}^{(5)}}=8.4266 \overline{\lambda_{5}^{(5)}}=10.4020 .
\end{aligned}
$$




\section{Conflict of Interests}

The authors declare that there is no conflict of interests regarding the publication of this paper.

\section{Acknowledgments}

This work is supported by the Natural Science Foundation of Jiangxi, China (nos. 20114BAB201015, 20122BAB201013, and 20132BAB201056), and Scientific and Technological Project of Jiangxi Education Office, China (no. KJLD13093).

\section{References}

[1] J. Peng, X.-Y. Hu, and L. Zhang, "Two inverse eigenvalue problems for a special kind of matrices," Linear Algebra and Its Applications, vol. 416, no. 2-3, pp. 336-347, 2006.

[2] H. Pickmann, J. Egaña, and R. L. Soto, "Extremal inverse eigenvalue problem for bordered diagonal matrices," Linear Algebra and its Applications, vol. 427, no. 2-3, pp. 256-271, 2007.

[3] A. M. Nazari and Z. Beiranvand, "The inverse eigenvalue problem for symmetric quasi anti-bidiagonal matrices," Applied Mathematics and Computation, vol. 217, no. 23, pp. 9526-9531, 2011.

[4] H. Pickmann, R. L. Soto, J. Egaña, and M. Salas, "An inverse eigenvalue problem for symmetrical tridiagonal matrices," Computers \& Mathematics with Applications, vol. 54, no. 5, pp. 699-708, 2007.

[5] D. Boley and G. H. Golub, "A survey of matrix inverse eigenvalue problems," Inverse Problems, vol. 3, no. 4, pp. 595-622, 1987.

[6] M. T. Chu and G. H. Golub, Inverse Eigenvalue Problems: Theory, Algorithms, and Applications, Oxford University Press, New York, NY, USA, 2005.

[7] J. C. Egaña, N. M. Kuhl, and L. C. Santos, "An inverse eigenvalue method for frequency isolation in spring-mass systems," Numerical Linear Algebra with Applications, vol. 9, no. 1, pp. 6579, 2002.

[8] G. M. L. Gladwell, "Inverse problems in vibration," Applied Mechanics Reviews, vol. 39, 1986.

[9] O. H. Hald, "Inverse eigenvalue problems for Jacobi matrices," Linear Algebra and Its Applications, vol. 14, no. 1, pp. 63-85, 1976.

[10] X. Y. Hu, L. Zhang, and Z. Y. Peng, "The construction of a Jacobi matrix from its defective eigen-pair and a principal submatrix," Mathematica Numerica Sinica, vol. 22, no. 3, pp. 345-354, 2000.

[11] A. P. Liao and Z. Z. Bai, "Construction of positive definite Jacobian matrices from two eigenpairs," Journal on Numerical Methods and Computer Applications, vol. 23, no. 2, pp. 131-138, 2002 (Chinese).

[12] L. Lu and M. K. Ng, "On sufficient and necessary conditions for the Jacobi matrix inverse eigenvalue problem," Numerische Mathematik, vol. 98, no. 1, pp. 167-176, 2004. 


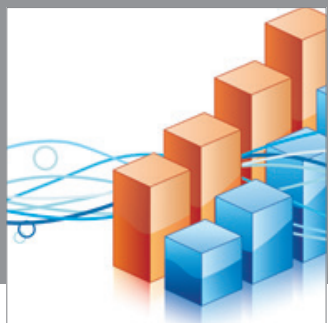

Advances in

Operations Research

mansans

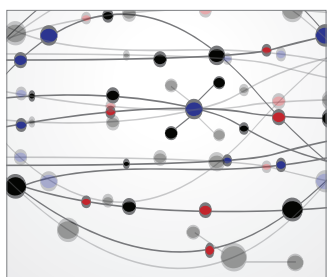

The Scientific World Journal
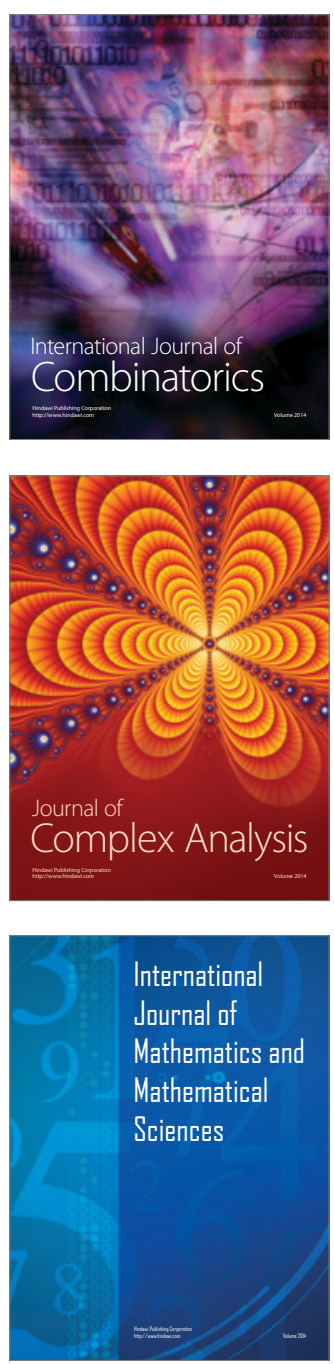
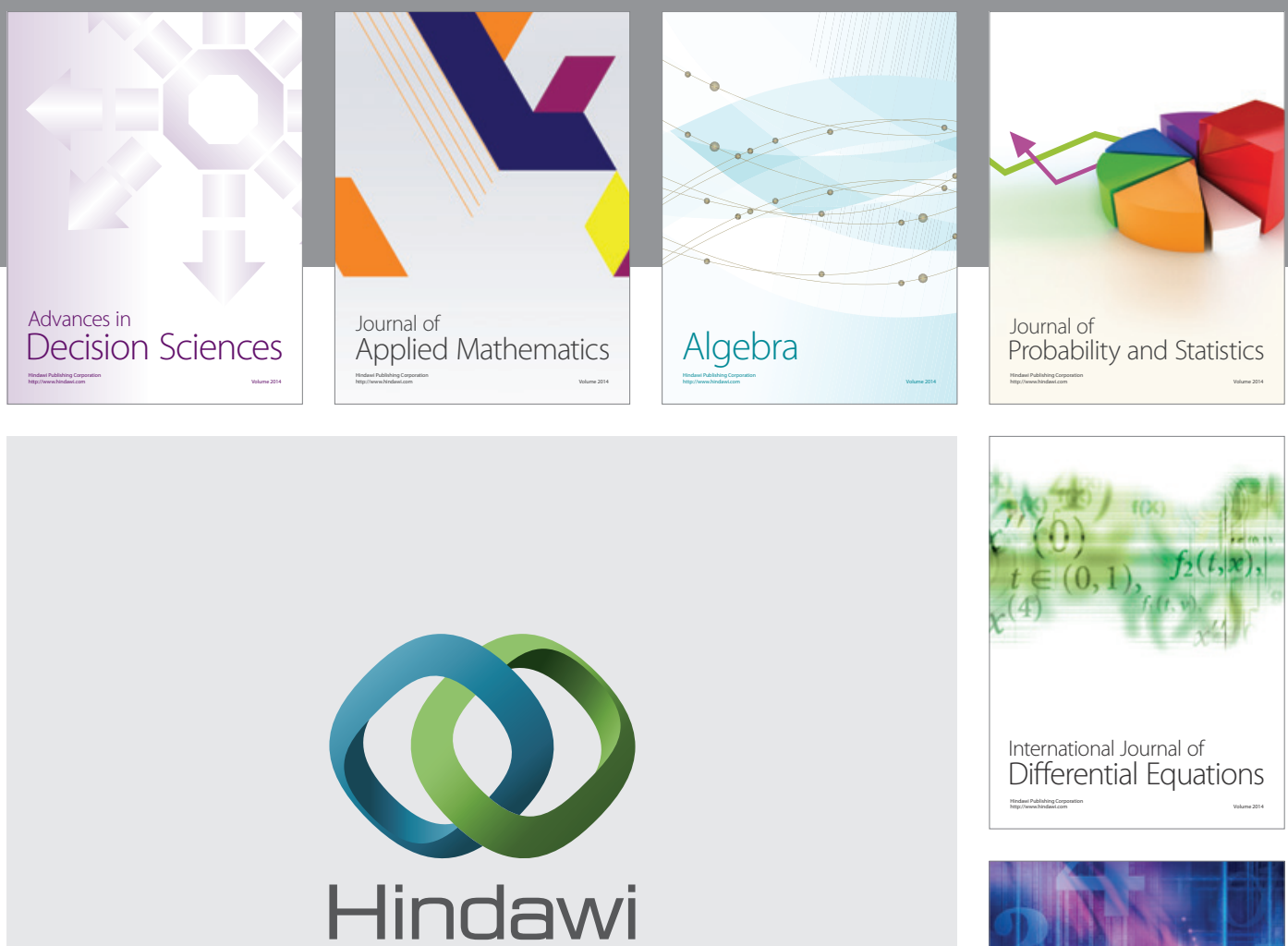

Submit your manuscripts at http://www.hindawi.com
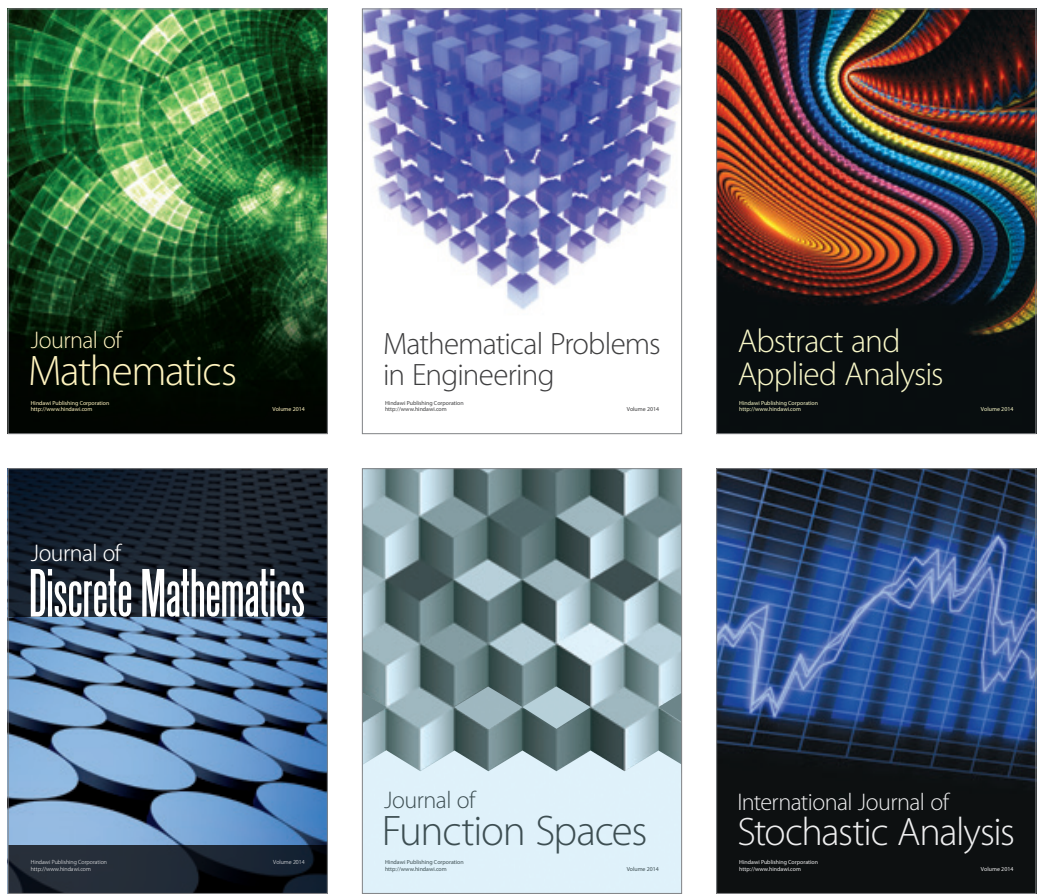

Journal of

Function Spaces

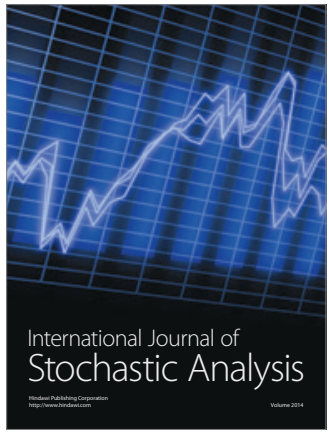

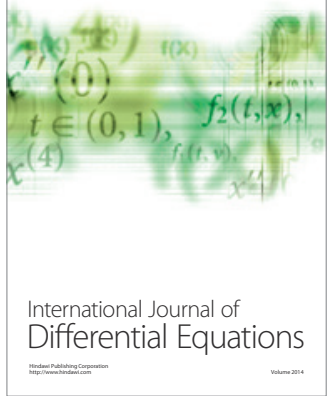
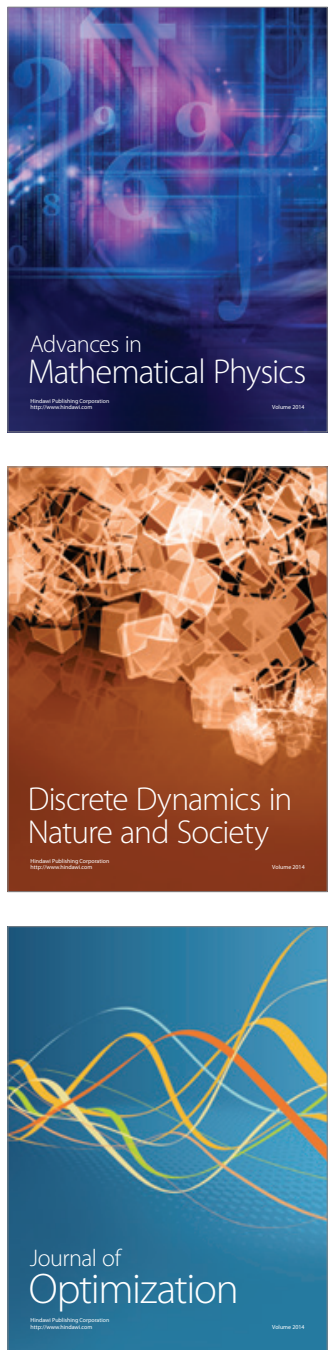